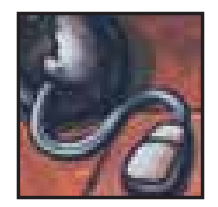

Web Review

\title{
Benchmarking Web Sites
}

by Mike Moralis

B

enchmarking" was originally a surveying term. In that context, it refers to a reference point for mapping purposes. It entered the realm of business management jargon in the 1980 s through the work of Robert C. Camp at Xerox Corporation, where he developed the concept and applied it to performance measurement and identification of best practices.

In 1989, Camp wrote the best-selling Benchmarking: The Search for Industry Best Practices that Lead to Superior Performance. In it, he describes benchmarking as a continuous process of measuring products, services and practices against leading competitors or known leaders. Benchmarking rapidly became part of the toolkit that managers and consultants brought to bear on issues such as competitiveness and quality improvement. In the 1990 s, driven by pressures to demonstrate quality and cost-effectiveness, improve accountability, and identify and meet changing expectations, the healthcare sector began to embrace these concepts, although not for the first time. In the 19th century, Florence Nightingale attracted attention, and achieved some reforms, by publishing mortality rates for London hospitals.

As an industry, healthcare has suffered from a wealth of data and a shortage of information. Computerization has facilitated efforts to move toward evidence-based decision-making at both the clinical and management levels by making data collection faster and easier. The development of the "balanced score-

\section{Benchmarking as a continuous process of measuring}

\author{
products, services and practices against leading
}

\section{competitors or known leaders.}

card" concept, led by Robert Kaplan and David Norton, was an effort to move beyond financial indicators and determine what aspects of performance can be measured and what should be measured for strategic management purposes. They ended up with a methodology for doing that, as well as for "translating strategy into action," as their book The Balanced Scorecard is subtitled. Healthcare organizations investigated the concept as a potentially useful tool, and some efforts are being made to apply it.

Recommended web sites:

\section{The Benchmarking Exchange} http://www.benchnet.com/

The Benchmarking Exchange is an international and user-friendly repository of information about benchmarking. There is a membership fee, but the public area includes a searchable list of about 700 members, which includes a number of Canadian organizations, some in the healthcare sector, but no hospitals. The web site observes that it is only natural for organizations engaged in benchmarking to want to compare their efforts in that area to those of similar organizations. Membership provides the ability to do literature searches.

\section{Serving the American Public: Best Practices in Performance Measurement}

http://www.npr.gov/library/papers/ benchmrk/nprbook.html

In 1993, U.S. President Bill Clinton and Vice President al Gore implemented the National Performance Review as part of their contribution to "reinventing government." One of the initiatives was benchmarking of "best-in-class" organizations in the public and private sectors. Two reports on best practices were produced in 1997, one on CustomerDriven Strategic Planning, and the other on Best Practices in Performance Measurement. The latter included U.S. federal agencies, local governments, and some Canadian government agencies including Atomic Energy Canada Ltd., Industry Canada, Treasury Board, the St. Lawrence Seaway Authority, and the federal land registry. The 36-page report is a primer on how to succeed in benchmarking, and includes a glossary and contact phone numbers and e-mail addresses to help reach participants.

\section{National Forum on Health}

http://wwwnfh.hc-sc.gc.ca

Although the National Forum on Health 
only wrapped up its work in 1997, some people may have already forgotten its call for the development of a national set of performance indicators and benchmarks for healthcare. The final report of the Working Group on Striking a Balance recommended: "That provincial/territorial agencies together with a national agency give priority to developing a set of indicators and benchmarks to be used by all jurisdictions for assessment of the state of access to appropriate health services, and as part of their public reporting function, collect and make this information public at regular intervals."

\section{Capital Health Authority (Alberta)}

http://www.cha.ab.ca/cha/repo9708

As part of province-wide efforts to track rapid changes and meet new standards of accountability, Alberta's healthcare system is developing a variety of measures for comparison, some of them against provincial targets. The performance indicators developed by the Capital Health Authority in Edmonton are among the most comprehensive and readable. The measures fall into five categories: appropriateness of services provided (including hospital bed utilization, immunization rates and caesarean sections), satisfaction of people with the services they receive, quality of care (including selected followups and re-admissions), access to health services (including selected waiting times), and financial and utilization (including spending on administration and a comparison of spending on institutions versus community services). The report goes to the region's board semiannually, and then becomes public.

\section{The Maryland Hospital AsSOCIaTION QUALITY INDICATOR Project ${ }^{R}$}

http://www.qiproject.org

The Maryland Hospital Association Quality Indicator Project $\AA$ began in 1985 as a voluntary, pilot appliedresearch project involving seven hospi- tals with a straightforward mission: to develop valid indicators that will assist participants in understanding and improving their performance. Today, more than 1100 hospital across the U.S. and in the U.K. are participants through multi-hospital systems or hospital associations. They use the project's 10 in-patient indicators, as well as five for ambulatory care, seven for psychiatric care and five for long-term care. There is an annual participation fee, which entitles participants to the project's software, materials and services including consultation.

\section{New York State Department of HeALTH}

http://www.health.state.ny.us

The New York State Department of Health has a comprehensive web site that includes pages with information for consumers, providers and researchers. New York first began publishing hospital-specific mortality data for cardiac surgery in 1989. The consumer web page includes 1996 and 1997 reports on coronary artery bypass surgery with hospital-and physician-specific riskadjusted mortality rates, and a hospital-specific report on angioplasty. There is also a database, searchable by name, of monthly professional misconduct and physician disciplinary actions.

\section{Michigan Health \& Hospital Association}

http://www.mha.org

The Michigan Health \& Hospital Association releases a state-wide performance report annually "to help Michigan patients, purchasers and providers become better informed about healthcare." Two editions have been published, and the third is scheduled for release this spring. The 1997 report covers hospital-specific expected and actual performance for eight types of medical conditions and surgical procedures that occur frequently. The report is based on records submitted voluntari-

\section{Check Them Out!}
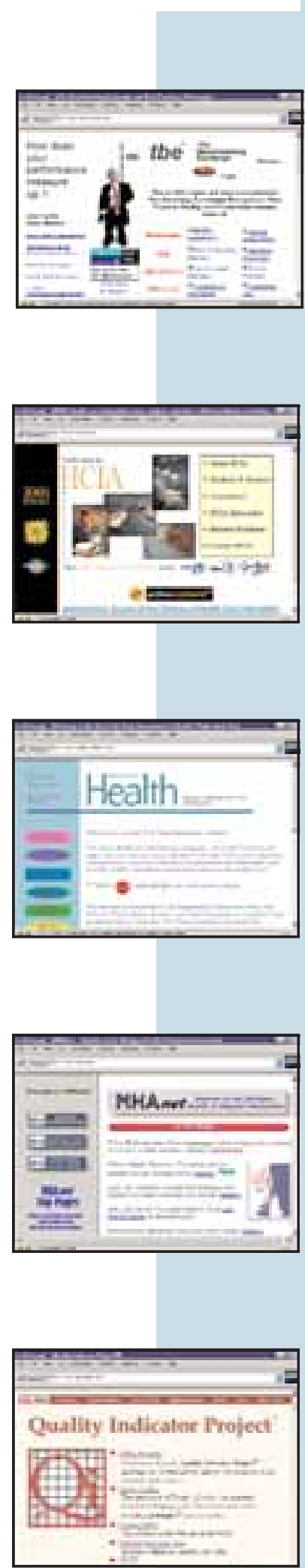
ly by all hospitals reporting to the Michigan Inpatient Data Base (MIDB), amounting to 1.3 million in-patient discharges in 1995.

\section{Cleveland Health Quality Choice Program}

http://clevnet.cpl.org/CHQC

To quote the Greater Cleveland Consumer Report on Hospital Performance: "In 1989, businesses, hospitals and physicians in a fourcounty area surrounding Cleveland, Ohio came together to form a unique, voluntary partnership for the purposes of instituting a healthcare marketreform program." The program's goal was to develop a system to "reliably and objectively measure and compare patient outcomes and patient satisfaction as an indication of quality..."

Twice a year, it publishes excellent reports of about 50 pages in length, including explanatory text and detailed charts. The reports are posted on the web site, and are now in PDF format.

\section{Australian Institute of Health and Welfare - Health Sector Performance Indicators}

http://www.aihw.gov.au/publications/ h_online/indic97/indic.htm

In 1994, at an Australian Health Ministers' Conference, a Benchmark Working Group was formed, consisting of the Australian Commonwealth, State and Territory health authority officers, the Australian Hospitals Association and the Australian Institute of Health and Welfare. The group developed a set of hospital performance indicators addressing efficiency, productivity, quality and access, and published a report in 1997. The First National Report on Health Sector Performance Indicators: Public Hospitals -- The State of Play runs to 90 pages plus appendices, and is available on the web site as a series of PDF files. It includes enough contextual information to constitute a primer on Australia's healthcare system, in addition to providing detailed charts, tables and explanation of various methodologies.

\section{HCIA INC.}

\section{http://www.hcia.com}

It's natural that consulting firms would be interested in promoting their expertise in benchmarking. HCIA says it has the largest healthcare database in the industry, with more than 325 million patient discharge records. HCIA and Mercer Health Care Provider Consulting produce an annual study comparing the 100 top-performing acute care hospitals in the Unites States against 100 benchmarks, broken down into five peer groups. Among the tantalizing findings of the report for 1997: if all acute care hospitals performed at the benchmark level, expenses would decline by an aggregate $\$ 24.5$ billion a year; and average lengths of stay would decrease by nearly half a day. While some information is available on the web site, the catch is that there's a $\$ 700$ (U.S.) fee for the detailed report, although an abstract that lists the top performers and explains the methodology is available for $\$ 80$. Ie

Mike Moralis is a Health Policy Analyst at the Ontario Hospital Association and a former journalist.

\section{ERRATA}

In Hospital Quarterly, vol. 1, no. 2:

Earl Berger, Associate Director of Policy Planning

at the HayGroup, was incorrectly identified.

A Working Paper Series, which appears on the Centre for Health Economics and Policy Analysis web site, was incorrectly attributed to the Institute for Clinical Evaluative Sciences in Ontario.

The legend for the graph related to laparoscopic cholecystectomies in the Quarterly Index (page 68) should have read "Rate/100,000 population.

\section{Sanofi FOR POSITION ONLY from prev issue}

